\title{
DEFORMING TESSELLATIONS FOR THE SEGMENTATION OF CELL AGGREGATES
}

\author{
Anaïs Badoual, Aymeric Galan, Daniel Sage, and Michael Unser \\ Biomedical Imaging Group, École polytechnique fédérale de Lausanne (EPFL), Switzerland
}

\begin{abstract}
We present a new active contour to segment cell aggregates. We describe it by a smooth tessellation that is attracted toward the cell membranes. Our approach relies on subdivision schemes that are tightly linked to the theory of wavelets. The shape is encoded by control points grouped in tiles. The smooth and continuously defined boundary of each tile is generated by recursively applying a refinement process to its control points. We deform the smooth tessellation in a global manner using a ridge-based energy that we have designed for that purpose. By construction, cells are segmented without overlap and the tessellation structure is maintained even on dim membranes. Leakage, which afflicts usual image-processing methods (e.g., watershed), is thus prevented. We validate our framework on both synthetic and real microscopy images, showing that the proposed method is robust to membrane gaps and to high levels of noise.
\end{abstract}

Index Terms-Active contour, C. elegans, cell aggregate, Deslauriers-Dubuc, segmentation, subdivision, tessellation.

\section{INTRODUCTION}

We address the segmentation of the cell aggregates that appear in images of several biological specimen such as C. elegans embryo or cornea endothelium (Figures 1 and 8 ). This task is challenging because of the proximity of the cells and the presence of gaps in the membranes.

Over the past decade, automated methods were proposed for the segmentation of cell membranes, including intensity thresholding, morphological operations, Voronoi-based methods [1], labeling procedures [2], or watershed transform [3-7]. Those methods have three main limitations. First, they suffer from leakage in case of dimmed membranes. Second, they are sensitive to noise and might result in over-segmentation. Third, it is not easy to introduce prior knowledge to improve the accuracy of the segmentation [8]. Recent approaches are based on the detection of membrane patterns coupled with graph-cut [9], or on deep learning [10]. If these methods are better suited for incorporating prior knowledge, they do not allow for easy and user-friendly interaction. Moreover, they may provide non-continuous cell boundaries, which complicates the extraction of quantitative measurements. Topology adaptive methods, such as level sets or T-snakes [11,12], are not required in this context as the topological structure of the cell aggregate is generally known.

Parametric active contours-a.k.a. snakes-are popular models for the interactive segmentation of bioimages [13-17]. They consist in a curve that evolves from an initial position toward the boundary of the object of interest through the minimization of an energy term $[16,18]$. The underlying shape has a continuous representation in terms of basis functions [16], which facilitates the incorporation

This work was funded by the Swiss National Science Foundation under Grant 200020-162343.

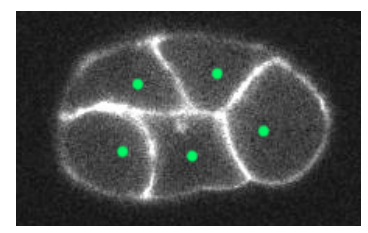

(a) Seed points.

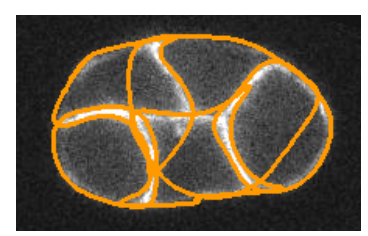

(c) Five classical snakes.

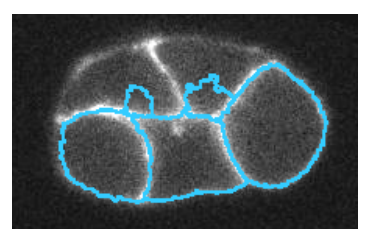

(b) Watershed.

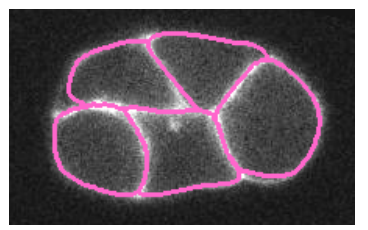

(d) Active tessellation, $J=0.98$.
Fig. 1. Segmentation of a C. elegans embryo; Source: R. Jankele and P. Gönczy, EPFL. (a) Seed points used for the initialization of each method; (b) watershed method; (c) five classical snakes [16]; (d) active tessellation.

of prior knowledge. They are built to ensure continuity and smoothness, which prevents leakage. They are parametrized by only a few control points, which results in fast optimization and provides robustness to noise. However, parametric snakes are not well suited to segment objects that are close to each other: They might yield overlapping segmentations (Figure 1 (c)) as the snakes are optimized independently. In the literature, only few works regarding active contours address the segmentation of touching objects. Extension of traditional parametric snakes to track non-occluding objects that transiently touch each other are presented in $[19,20]$. These methods could only segment cell aggregates with thick membranes or non-touching cells. Networks of active contours were introduced in $[21,22]$. However, they involve many parameters (nodal points).

In this paper, we propose a new active contour with a geometric representation that keeps the advantages of parametric snakes while addressing globally the segmentation problem of cell aggregates. The model consists in a smooth tessellation, so-called active tessellation, that is globally deformed towards the cell membranes through the minimization of a suitable ridge-based energy. The smooth tessellation is encoded by a set of control points and generated through a geometric subdivision scheme, a computer-graphics tool for representation and modeling. By construction, the segmented tiles are non-overlapping and the tessellation structure bridges membrane gaps. After optimization, each cell of the aggregate can be individually extracted to compute statistical descriptors.

The main contributions of this work are: 1) the construction of a smooth tessellation to describe an active contour; 2) the derivation of an oriented ridge-based energy functional; 3 ) the implementation of the whole framework. We illustrate the benefits of the proposed active tessellation on real biological applications in the context of semi-interactive segmentation. 


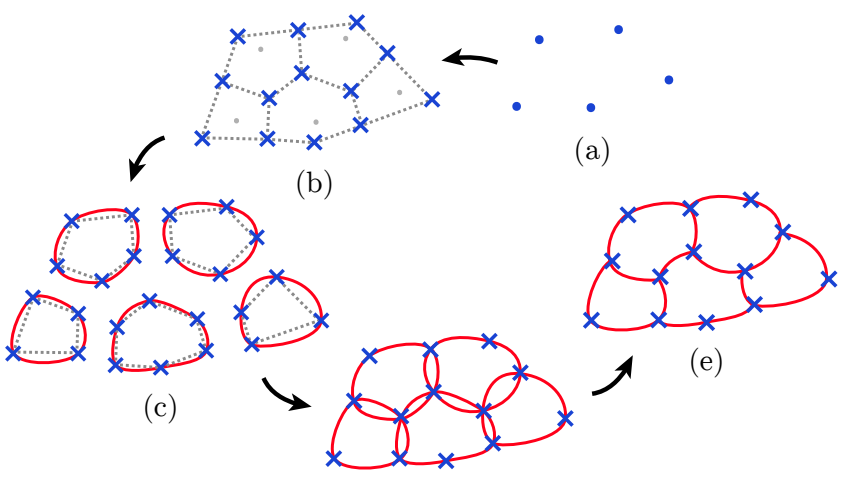

(d)

Fig. 2. Construction of the smooth tessellation. Given seed points (a), we first generate control points (blue crosses) grouped in tiles (b). Then, we specify the smooth boundary of each tile with a continuous closed curve by applying a subdivision scheme to its control points (c). Finally, we keep one of the common edges (d) to obtain the final smooth tessellation (e).

\section{CONSTRUCTION OF A SMOOTH TESSELLATION}

Our active contour model is a smooth tessellation, as described in Figure 2 (e). Its shape is parametrized by control points that are grouped in tiles (Figure 2 (b)). Each tile is associated with a closed curve that specifies its smooth boundary (Figure 2 (c)). This smooth curve is generated from the control points via a subdivision scheme (Section 2.1). The smooth tessellation is the union of the closed curve of each tile. As two control points that belong to two adjacent tiles are connected by two continuous edges (Figure 2 (d)), we keep only the edge of the largest tile (Figure 2 (e)). The remaining edges are then optimized by fitting them to the image data via energy optimization (Section 3).

\subsection{A Tiling Made of Closed Subdivision Curves}

A subdivision scheme allows one to describe a contour by an initial set of control points which, by the iterative application of a refinement rule, becomes continuous in the limit [23-26]. The smoothness and geometric-reproduction properties of the limit curve depend on the particular choice of the subdivision mask. We use this powerful mathematical method to generate the closed curve of each tile.

\subsubsection{Notations}

We represent by $\mathbf{p}_{(k)}[\cdot]$ a $\left(2^{k} M\right)$-periodic sequence of points $\mathbf{p}_{(k)}[m]=\left(p_{1_{(k)}}[m], p_{2_{(k)}}[m]\right)$, indexed by $m \in \mathbb{Z}$, where $p_{1_{(k)}}$ and $p_{2(k)}$ are the coordinates. The discrete convolution of $\mathbf{p}_{(k)}[\cdot]$ with a scalar mask $h[\cdot]$ is defined as $\left(h * \mathbf{p}_{(k)}\right)[m]=$ $\sum_{n \in \mathbb{Z}} h[m-n] \mathbf{p}_{(k)}[n]$.

\subsubsection{Closed Subdivision Curves}

Let $\mathbf{p}_{(0)}$ be the $M$-periodic sequence that contains the control points of a tile. The closed curve associated to this tile is generated as follows (Figure 3): We apply a refinement rule to $\mathbf{p}_{(0)}$ to double the number of points. We repeat this operation $k$ times to obtain the $\left(2^{k} M\right)$-periodic sequence of subdivision points $\mathbf{p}_{(k)}$. When $k \rightarrow \infty$, the set of points converges to the continuous curve $\mathbf{r}=\left(r_{1}, r_{2}\right)$ with $r_{1}, r_{2} \in \mathcal{C}^{1}$. In practice, $k=6$ iterations are enough for the contour points to be sufficiently dense. The subdivision points at the $k$ th iteration $(k \geq 1)$ are directly obtained from $\mathbf{p}_{(0)}$ by

$$
\mathbf{p}_{(k)}=h_{0 \rightarrow k} * \mathbf{p}_{(0) \uparrow_{2} k},
$$

where $\uparrow_{2^{k}}$ denotes an upsampling by a factor of $2^{k}, h_{0 \rightarrow k}=$ $h_{\uparrow_{2} k-1} * h_{\uparrow_{2} k-2} * \cdots * h_{\uparrow_{2}} * h$ [17], and $h$ is the subdivision mask whose z-transform is

$$
H(z)=-\frac{1}{16} z^{-3}+\frac{9}{16} z^{-1}+1+\frac{9}{16} z-\frac{1}{16} z^{3} .
$$

This subdivision mask corresponds to the convergent subdivision scheme introduced by Deslauriers and Dubuc in [27], which generates $\mathcal{C}^{1}$-continuous functions and reproduces polynomials up to degree 3 [27]. This scheme is interpolating [27, 28], which means that the subdivision points, at each level of the process, interpolate the limit curve $\mathbf{r}$. We thus have that

$$
\left.\mathbf{r}(t)\right|_{t=\frac{m}{2^{k}}}=\mathbf{p}_{(k)}[m] .
$$

This is an advantage when user interaction is involved, as it facilitates the editing of the curve. Moreover, it is affine invariant, so that the geometry of the limit curve changes in synchrony with any affine transformation that would be applied to the initial set of control points. This is an important property that facilitates the design of active contours.

\section{AN ORIENTED RIDGE-BASED ENERGY}

The energy functional $E$ drives the deformation of the smooth tessellation. Usually, this energy is divided into an internal energy, which ensures the smoothness of the curve, and an external energy, which is purely data driven. In our formulation, the smoothness of the curve is ensured by the Deslauriers-Dubuc subdivision scheme that produces $C^{1}$-continuous curve. Since we want the external term to attract the smooth tessellation toward the cell membranes, we reduce $E$ to a ridge-based energy term.

To detect ridges, a common approach is to compute the Hessian matrix $\mathbf{U}$ at location $\mathbf{x}=\left(x_{1}, x_{2}\right)$ as

$$
\mathbf{U}=\left(\begin{array}{ll}
\frac{\partial^{2} u\left(x_{1}, x_{2}\right)}{\partial x_{1}^{2}} & \frac{\partial^{2} u\left(x_{1}, x_{2}\right)}{\partial x_{1} \partial x_{2}} \\
\frac{\partial^{2} u\left(x_{1}, x_{2}\right)}{\partial x_{2} \partial x_{1}} & \frac{\partial^{2} u\left(x_{1}, x_{2}\right)}{\partial x_{2}^{2}}
\end{array}\right),
$$

where $u\left(x_{1}, x_{2}\right)=f\left(x_{1}, x_{2}\right) *\left(g\left(x_{1}\right) g\left(x_{2}\right)\right)$ with $f$ the input image and $g\left(x_{i}\right)=\frac{1}{\sigma \sqrt{2 \pi}} \mathrm{e}^{\frac{-\left(x_{i}\right)^{2}}{2 \sigma^{2}}}$ for $i=1,2$. High values of the standard deviation $\sigma \in \mathbb{R}$ increase the basin of attraction of the active contour. We define the ridge strength at location $\mathrm{x}$ by

$$
\xi(\mathbf{x})=\sqrt{\left|\lambda_{\min }\right|} \cdot \sqrt{\left|\lambda_{\min }-\lambda_{\max }\right|},
$$

where $\lambda_{\min }$ and $\lambda_{\max }$ are the minimum and maximum eigenvalues of $\mathbf{U}$, respectively. On the ridge, the ridge strength is maximum and the eigenvector $\mathbf{v}_{\min }$ is normal to the ridge.

Let $\mathbf{c}$ be the control points of the active tessellation. They generates a set of $N$ points $\mathbf{p}_{\text {tess }}=\mathbf{p}_{\text {tess }}(\mathbf{c})$ that delineates the smooth tessellation (Figure 3 ). The oriented ridge-based energy that we propose is

$$
\begin{aligned}
E\left(f, \mathbf{p}_{\text {tess }}\right)= & -\frac{1}{N} \sum_{m=0}^{N-1} \xi\left(\mathbf{p}_{\text {tess }}[m]\right) \\
& \times \frac{\left|\mathbf{v}_{\min }^{\top}\left(\mathbf{p}_{\text {tess }}[m]\right) \cdot \mathbf{n}\left(\mathbf{p}_{\text {tess }}[m]\right)\right|}{\left\|\mathbf{v}_{\min }\left(\mathbf{p}_{\text {tess }}[m]\right)\right\|\left\|\mathbf{n}\left(\mathbf{p}_{\text {tess }}[m]\right)\right\|},
\end{aligned}
$$




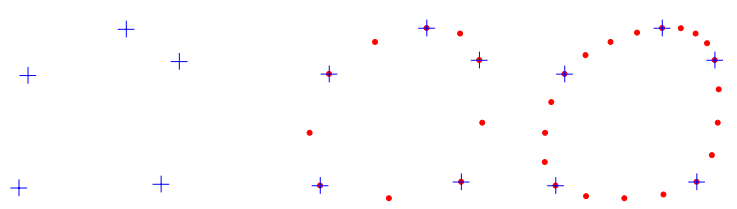

(a) $\mathbf{p}_{0}=\mathbf{c}$
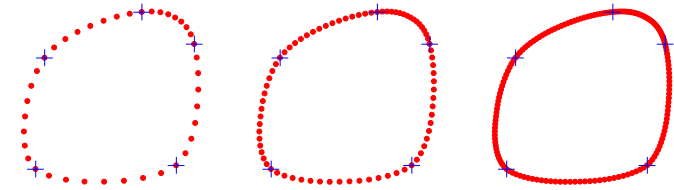

(b) Subdivision steps.

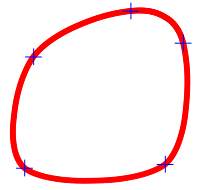

(c) $\mathbf{p}_{6}=\mathbf{p}_{6}(\mathbf{c})$

Fig. 3. Interpolating Deslauriers-Dubuc subdivision scheme. (a) Control points $\mathbf{p}_{0}$; (b) subdivision steps that converge to the continuous curve (c) which is encoded by the five control points $\mathbf{p}_{0}$ (blue crosses).

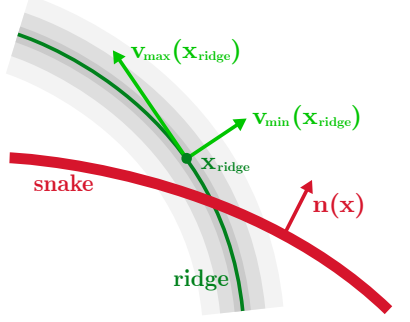

Fig. 4. Ridge feature. When minimizing the ridge-based energy (6), the active tessellation (red line) tends to align with the center of the ridge (green line).

where $\mathbf{n}\left(\mathbf{p}_{\text {tess }}[m]\right)$ is the normal vector at the $m$ th point. The energy (6) is minimal when the vectors $\mathbf{v}_{\min }$ and $\mathbf{n}$ are aligned and when the tessellation lies on the ridge (Figure 4). The optimization is computed as a function of the control points c. The optimum is obtained as

$$
\mathbf{c}_{\mathrm{opt}}=\underset{\mathbf{c}}{\arg \min } E\left(f, \mathbf{p}_{\text {tess }}(\mathbf{c})\right)
$$

\section{IMPLEMENTATION}

We implemented the proposed framework as a user-friendly plugin for the bioimaging platform Icy [29]. ${ }^{1}$

We initialize the active tessellation as follows: We construct a Voronoi diagram around seed points and then apply a mask to shrink the Voronoi tiles in the neighborhood of the cell aggregate. This mask is obtained by constructing the convex hull of the seed points. The vertices of the resulting tiles are the control points of the active tessellation. Note that the seed points are manually specified through the interface or automatically detected from a provided image of the cells' nuclei.

We follow a coarse-to-fine optimization strategy. We first optimize the active tessellation made of the few control points of the Voronoi tiling using a Powell-like line-search method. We obtain a rough segmentation that is less likely to be stuck in local minima. We then double the number of control points to increase the flexibility of the active tessellation and we optimize it once again. This strategy makes our active contour less sensitive to initialization.

\section{EXPERIMENTS AND VALIDATION}

We perform three experiments to evaluate the performance of our active tessellation. We first investigate its robustness with respect to noise and dim membranes on synthetic data. Then, we illustrate applications on real data.

\footnotetext{
${ }^{1}$ The source code and a demo of the plugin are available at http://bigwww.epfl.ch/demo/active-tessellation/, as of October 2018.
}

\subsection{Synthetic Data}

We compare our approach in term of accuracy against the watershed method [30] implemented by I. Arganda-Carreras and D. Legland for the bioimage platform Fiji ${ }^{2}$. We use the Jaccard index $(J)$ to measure the similarity between the segmentation result $\Omega$ and the corresponding ground truth $\Omega_{\mathrm{GT}}$. It is defined as $0 \leq J=\frac{\left|\Omega \cap \Omega_{\mathrm{GT}}\right|}{\left|\Omega \cup \Omega_{\mathrm{GT}}\right|} \leq 1$. In the experiments, we compute the Jaccard index for each cell and take the average. It is this average value that we refer to as Jaccard index in the next Sections.

We created a test image that simulates the fluorescence microscopy of a $C$. elegans embryo with 5 cells (Figure 5 (a)). We use the same seed points to initialize the two methods. The initial configuration of the active tessellation is illustrated in Figure 5 (a). Its initial similarity with the ground truth corresponds to $J=0.64$.

\subsubsection{Robustness with Respect to Noise}

We corrupted the test image by different levels of additive Gaussian noise (20 realizations per level of noise, Figure 5 (d)). The signal-tonoise ratio (SNR) corresponding to a given noise level and Jaccard index were computed. The SNR that we use is the ratio of the mean value of the signal and the standard deviation of the noise. The results are given in Figure 6 and illustrated in Figures 5 (e) and (f). We observe that the active tessellation is robust with respect to noise since it is able to give a proper segmentation outcome even for low SNRs. On the contrary, the accuracy of the watershed method degrades significantly for a SNR below 2.4 .

\subsubsection{Robustness with Respect to Dim Membranes}

We progressively dimmed the fluorescence signal on the membranes of the test image (Figure $5(\mathrm{~g})$ ). We computed the Jaccard index as a function of the information-loss percentage. This dimming percentage corresponds to the ratio of the mean intensity on the membrane of the test image over the one of the corrupted image. The resulting plot is given in Figure 7 and we illustrate results in Figures 5 (h) and (i). The active tessellation accurately segments the cells until $49 \%$ of information loss while the watershed method can tolerate no more than $15 \%$ of information loss, then it quickly decreases. As this model is only based on intensity, it leaks through dim membranes. Due to the structure and smoothness of the active tessellation, the proposed framework does not suffer from leakage.

\subsection{Segmentation on Real Data}

We applied our active tessellation on real biomedical images. These images are challenging because of the presence of noise and gaps in the membranes. For each segmentation, the initial configuration of

\footnotetext{
${ }^{2}$ The source code is available at https://imagej.net/Classic_Watershed.
} 


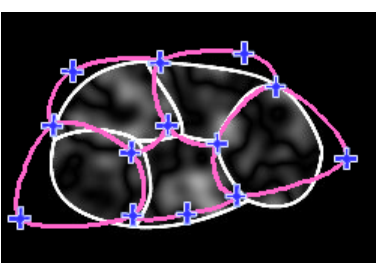

(a) Test image.

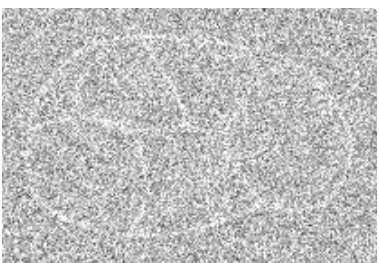

(d) Noisy image.

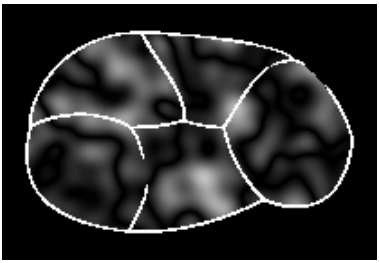

(g) Image with dimmed membranes.

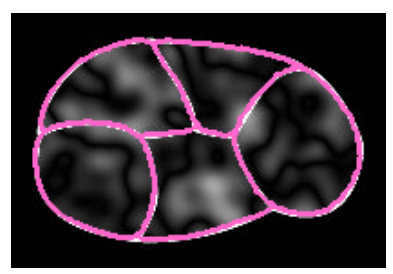

(b) Active tessellation, $J=0.98$.

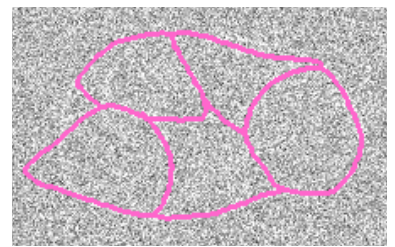

(e) Active tessellation, $J=0.90$.

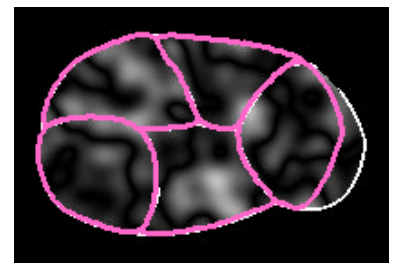

(h) Active tessellation, $J=0.94$.

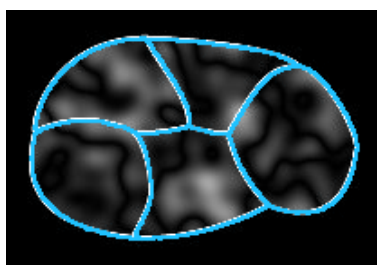

(c) Watershed, $J=1.0$.

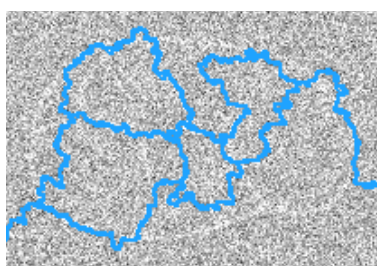

(f) Watershed, $J=0.46$.

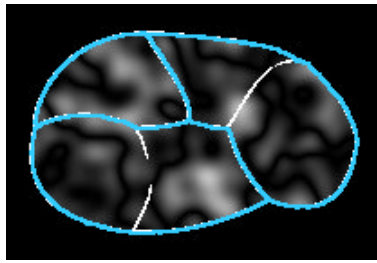

(i) Watershed, $J=0.44$

Fig. 5. Segmentation outcomes. (a)-(c) Test images. (a) Initial configuration of the active tessellation; (d)-(f) noisy data with SNR=0.81; (g)-(i) image with $23.95 \%$ of membrane information loss.

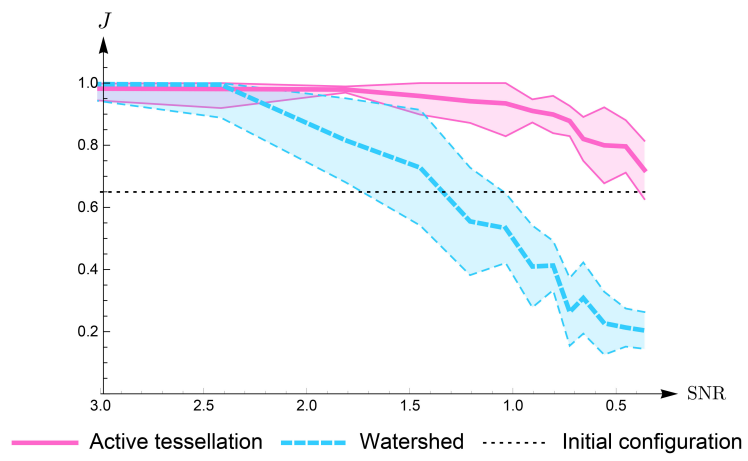

Fig. 6. Segmentation of noisy data. Evolution of the Jaccard index as a function of the SNR. Filled area: standard deviation across the 20 realizations.

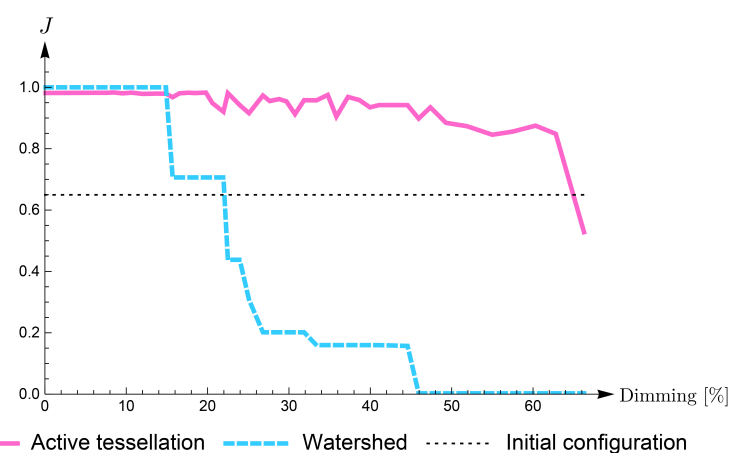

Fig. 7. Evolution of the Jaccard index as a function of the dimming percentage.

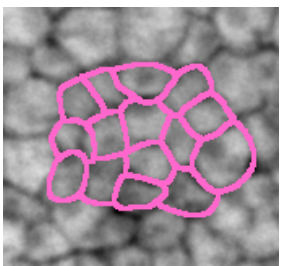

(a) $J=0.86$.

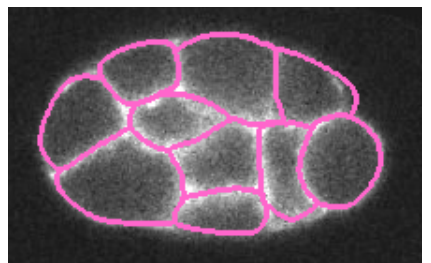

(b) $J=0.95$.
Fig. 8. Cell segmentation of (a) cornea endothelium; (b) C. elegans embryo in a light-sheet fluorescence-microscopy image. Source: R. Jankele and P. Gönczy, EPFL.

the active tessellation has 2.6 control points per cell in average. We compute the Jaccard index of each outcome considering a manual segmentation as ground truth. The results obtained are satisfactory in most cases (Figures 1 and 8).

\section{CONCLUSION}

We have presented a new subdivision-based active contour for the segmentation of cell aggregates. We have modeled the active contour by a smooth tessellation and designed an oriented-ridge-based energy term to efficiently attract the curve toward the membranes. The tessellation structure prevents from overlapping segmentation of the cells and from leakage issues. Moreover, each cell of the segmentation outcome can be easily extracted as a continuous closed curve making possible the computation of cell metrics. We have demonstrated the robustness of our method under noisy conditions and to dim membranes. We have also illustrated its behavior on real bioimages. 


\section{REFERENCES}

[1] T. R. Jones, A. Carpenter, and P. Golland, "Voronoi-based segmentation of cells on image manifolds," in International Workshop on Computer Vision for Biomedical Image Applications, Beijing, China, October 21, 2005, Springer, pp. 535-543.

[2] E. Ficarra, S. Di Cataldo, A. Acquaviva, and E. Macii, "Automated segmentation of cells with IHC membrane staining," IEEE Transactions on Biomedical Engineering, vol. 58, no. 5, pp. 1421-1429, May 2011.

[3] F. Meyer, "Topographic distance and watershed lines," Signal Processing, vol. 38, no. 1, pp. 113-125, July 1994.

[4] Y. Azuma and S. Onami, "Biologically constrained optimization based cell membrane segmentation in C. elegans embryos," BMC bioinformatics, vol. 18, no. 1, pp. 307, June 2017.

[5] K.R. Mosaliganti, R.R. Noche, F. Xiong, I.A. Swinburne, and S.G. Megason, "ACME: Automated cell morphology extractor for comprehensive reconstruction of cell membranes," PLoS Computational Biology, vol. 8, no. 12, pp. e1002780, December 2012.

[6] A. Chakraborty, M.M. Perales, G.V. Reddy, and A.K. RoyChowdhury, "Adaptive geometric tessellation for 3D reconstruction of anisotropically developing cells in multilayer tissues from sparse volumetric microscopy images," PLoS One, vol. 8, no. 8, pp. e67202, August 2013.

[7] J. Stegmaier, F. Amat, W.C. Lemon, K. McDole, Y. Wan, G. Teodoro, R. Mikut, and P.J. Keller, "Real-time threedimensional cell segmentation in large-scale microscopy data of developing embryos," Developmental Cell, vol. 36, no. 2, pp. 225-240, January 2016.

[8] D. S. Cheng, V. Murino, and M.A.T. Figueiredo, "Clustering under prior knowledge with application to image segmentation," in Advances in Neural Information Processing Systems, Vancouver, British Columbia, Canada, December 4-7, 2006, pp. 401-408.

[9] S. Dimopoulos, C.E. Mayer, F. Rudolf, and J. Stelling, "Accurate cell segmentation in microscopy images using membrane patterns," Bioinformatics, vol. 30, no. 18, pp. 2644-2651, September 2014.

[10] A. Fabijańska, "Segmentation of corneal endothelium images using a U-Net-based convolutional neural network," Artificial Intelligence in Medicine, vol. 88, pp. 1-13, June 2018.

[11] J.-P. Bergeest and K. Rohr, "Efficient globally optimal segmentation of cells in fluorescence microscopy images using level sets and convex energy functionals," Medical Image Analysis, vol. 16, no. 7, pp. 1436-1444, October 2012.

[12] T. McInerney and D. Terzopoulos, "T-snakes: Topology adaptive snakes," Medical Image Analysis, vol. 4, no. 2, pp. 73-91, June 2000.

[13] M.A.T. Figueiredo, J.M.N. Leitão, and A.K. Jain, "Unsupervised contour representation and estimation using B-splines and a minimum description length criterion," IEEE Transactions on Image Processing, vol. 9, no. 6, pp. 1075-1087, June 2000.

[14] B. Li and S.T. Acton, "Active contour external force using vector field convolution for image segmentation.," IEEE Transactions on Image Processing, vol. 16, no. 8, pp. 2096-2106, August 2007.
[15] R. Delgado-Gonzalo, V. Uhlmann, D. Schmitter, and M. Unser, "Snakes on a plane: A perfect snap for bioimage analysis," IEEE Signal Processing Magazine, vol. 32, no. 1, pp. 41-48, January 2015.

[16] R. Delgado-Gonzalo, P. Thévenaz, C.S. Seelamantula, and M. Unser, "Snakes with an ellipse-reproducing property," IEEE Transactions on Image Processing, vol. 21, no. 3, pp. 1258-1271, March 2012.

[17] A. Badoual, D. Schmitter, V. Uhlmann, and M. Unser, "Multiresolution subdivision snakes," IEEE Transactions on Image Processing, vol. 26, no. 3, pp. 1188-1201, March 2017.

[18] M. Jacob, T. Blu, and M. Unser, "Efficient energies and algorithms for parametric snakes," IEEE Transactions on Image Processing, vol. 13, no. 9, pp. 1231-1244, September 2004.

[19] C. Zimmer and J-C. Olivo-Marin, "Coupled parametric active contours," IEEE Transactions on Pattern Analysis and Machine Intelligence, vol. 27, no. 11, pp. 1838-1842, September 2005.

[20] Y. Deng, P. Coen, M. Sun, and J. W. Shaevitz, "Efficient multiple object tracking using mutually repulsive active membranes," PloS ONE, vol. 8, no. 6, pp. e65769, June 2013.

[21] P. Jasiobedzki, "Adaptive adjacency graphs," in Geometric Methods in Computer Vision II, San Diego, CA, United States, June 23, 1993, vol. 2031, pp. 294-304.

[22] M. Butenuth, "Segmentation of imagery using network snakes," Photogrammetrie Fernerkundung Geoinformation, vol. 2007, no. 1, pp. 7, May 2007.

[23] N. Dyn, "Subdivision schemes in CAGD," Advances in $\mathrm{Nu}$ merical Analysis, vol. 2, pp. 36-104, 1992.

[24] J. Warren and H. Weimer, Subdivision Methods for Geometric Design: A Constructive Approach, Morgan Kaufmann Publishers Inc., San Francisco, CA, USA, 1st edition, 2001.

[25] L. Romani, "From approximating subdivision schemes for exponential splines to high-performance interpolating algorithms," Journal of Computational and Applied Mathematics, vol. 224, no. 1, pp. 383-396, February 2009.

[26] M. Charina, C. Conti, and L. Romani, "Reproduction of exponential polynomials by multivariate non-stationary subdivision schemes with a general dilation matrix," Numerische Mathematik, vol. 127, no. 2, pp. 223-254, June 2014.

[27] S. Dubuc and G. Deslauriers, "Symmetric iterative interpolation processes," Constructive Approximation, vol. 5, no. 1, pp. 49-68, December 1989.

[28] C. Conti, L. Gemignani, and L. Romani, "From symmetric subdivision masks of Hurwitz type to interpolatory subdivision masks," Linear Algebra and its Applications, vol. 431, no. 10, pp. 1971-1987, October 2009.

[29] F. de Chaumont, S. Dallongeville, N. Chenouard, N. Hervé, S. Pop, T. Provoost, V. Meas-Yedid, P. Pankajakshan, T. Lecomte, Y. Le Montagner, T. Lagache, A. Dufour, and J.-C. Olivo-Marin, "Icy: An open bioimage informatics platform for extended reproducible research," Nature Methods, vol. 9, no. 7, pp. 690-696, July 2012.

[30] P. Soille and L.M. Vincent, "Determining watersheds in digital pictures via flooding simulations," in Proceedings of the SPIE Conference on Visual Communication and Image Processing, Lausanne, Switzerland, September 1, 1990, vol. 1360, pp. 240-251. 